\title{
Detection of Anaerobic Bacteria in Bronchoalveolar Lavage of Patients with Chronic Chest Lesion
}

\author{
Ahmed M. Wahba* ${ }^{* 1}$, Mona A. Abdel-Messih ${ }^{* 2}$, Randa S. Mohamed ${ }^{* 3}$ and Naglaa A. Radi ${ }^{* 1}$ \\ ${ }^{* 1}$ Department of Medical Microbiology, Beni Suef Faculty of Medicine Beni Suef University, Egypt \\ ${ }^{*}$ Department of Medical Microbiology, Cairo Faculty of Medicine Cairo University, Egypt \\ ${ }^{*}$ Department of Chest, Beni Suef Faculty of Medicine Beni Suef University, Egypt
}

\begin{abstract}
Anaerobic bacteria have important roles in the microbiota of humans and they are significant infectious agents involved in many pathological processes, both in immunocompetent and immunocompromised individuals. The objectives of this study is to detect anaerobic bacteria in bronchoalveolar lavage samples of patients with chronic chest Lesion in chest department in Beni -Suef university hospital.

Patients \& Methods : Each sample taken was placed immediately in previously prepared vacutainer containing thioglycolate media. Specimens from Thioglycollate broth after incubation were cultured on Brucella agar (Thermo Scientific ${ }^{\mathrm{TM}}$ Oxoid ${ }^{\mathrm{TM}}$ ) supplemented with 5\% lysed sheep blood, hemin and Vitamin K1 . The plates were incubated at $37^{\circ} \mathrm{c}$ for $48-27 \mathrm{~h}$ in anaerobic condition. Commercial Kits RapID ${ }^{\mathrm{TM}}$ ANA II System (Thermo Scientific ${ }^{\mathrm{TM}}$ Remel $^{\mathrm{TM}}$ ) used for the identification of anaerobic isolates. Antimicrobial susceptibility testing for anaerobic isolates were done using MIC Test Strip (Liofilchem, Italy).

Results : The isolated anaerobic organism from BAL specimens were 7 out of 25(28\%). The anaerobic isolates were Prevotella (3), Veillonella (2), Lactobacillus (1) and Propionibacterium (1).

Conclusion : Anaerobic bacteria are the most overlooked bacterial pathogens of the lower respiratory tract. They are often infrequently recovered from this site probably because of improper specimen collection and lack of appropriate anaerobic identification techniques.
\end{abstract}

Keywords : Anaerobe, Bronchoalveolar Lavage and Lung Abscess

\section{INTRODUCTION}

Anaerobic bacteria are relatively frequent pathogens in pulmonary infections that are associated with aspiration pneumonitis, lung abscess, necrotizing pneumonia and empyema [1].

Aspiration of oropharyngeal or gastric content, and severe periodontal or gingival disease predispose for anaerobic pleuropulmonary infection. The infection can progress from pneumonitis to necrotizing pneumonia, pulmonary abscess, and empyema [2].
Anaerobic bacteria are involved in all morbid conditions of respiratory infections, and the frequencies of their isolation are high in pneumonia and at the time of acute exacerbation of chronic lower airway infection. They are detected in high rate in lung abscess and pyothorax cases. Thus, anaerobic bacteria should not be neglected, because the frequency of their detection varies considerably among morbid conditions, and anaerobic bacteria do not merely drift down from the upper airway but also show major involvement in morbid conditions. It should be recognized that anaerobic bacteria are extensively involved not only in lung abscess and 
aspiration pneumonia, in which anaerobic infection has conventionally been indicated to participate, but also pneumonia and acute exacerbation of chronic lower airway infection [3].

\section{PATIENTS AND METHODS}

Subjects and data collection: The study was conducted on 25 patients admitted in the departments of chest in BeniSuef University Hospital .The patients were diagnosed as having chronic chest lesions ( 10 with lung abscess and 10 with lung tumor).The age ranged from 50 to 73 years old. From each patient a full history was taken as regards name, age and sex. All patients were advised to stop antimicrobial therapy at least 3 days before taken the samples. All procedures were done after taken a written concent from the patients.

\section{Sample processing and bacterial} identification : BAL were collected by bronchoscope. Specimens inoculated directly on previously prepared vacutainer containing Thioglycollate broth (Thermo Scientific ${ }^{\mathrm{TM}}$ Oxoid ${ }^{\mathrm{TM}}$ ) and incubated anaerobically for 48-72 hours at $37^{\circ}$ c.. Specimens from Thioglycollate broth after incubation were cultured on Brucella agar (Thermo Scientific ${ }^{\mathrm{TM}}$ Oxoid $^{\mathrm{TM}}$ ) supplemented with 5\% lysed sheep blood, hemin and Vitamin K1 .The plates were incubated at $37^{\circ} \mathrm{c}$ for $48-27 \mathrm{~h}$ in anaerobic jar with self-contained gas generating systems (Oxoid AnaeroGen, Thermo Scientific ${ }^{\mathrm{TM}}$ Oxoid $\left.^{\mathrm{TM}}\right)$. Commercial KitsRapID ${ }^{\mathrm{TM}}$ ANAII System (Thermo Scientific ${ }^{\mathrm{TM}}$ Remel $^{\mathrm{TM}}$ ) used for the identification of anaerobic isolates Procedure and interpretation of the method were followed according to manufacturer's instructions. (Thermo Scientific ${ }^{\mathrm{TM}}$ Remel $^{\mathrm{TM}}$ ).

Antibiotic susceptibility testing: Antimicrobial Susceptibility testing for anaerobic isolates were done using MIC Test Strip (Liofilchem, Italy). The interpretations were done according to CLSI-M100 guidelines [4]. The antibiotic used were: amoxicillin/clavlanic acid (AUG), clindamycin (CD), meropenem (MRP) and metronidazole (MTZ).

Medium used was Brucella agar (Thermo Scientific ${ }^{\mathrm{TM}}$ Oxoid ${ }^{\mathrm{TM}}$ ) supplemented with $5 \%$ lysed sheep blood, 5 $\mu \mathrm{g} / \mathrm{mL}$ hemin and $1 \mu \mathrm{g} / \mathrm{mL}$ Vitamin $\mathrm{K} 1$.

Interpretative MIC breakpoints of anaerobic species was done according to CLSI-M100 guidelines [4].

\section{RESULTS AND DISCUSSION}

The isolated anaerobic organism from BAL specimens were 7 out of 25(28\%). From lung abscess 5 cases yielded anaerobic organisms (50\%) while in cases of lung tumor only two anaerobic isolates (13.3\%) as shown in Table (1).

Table (1) Frequency of anaerobic organism isolated from broncho alveolar lavage (BAL)

\begin{tabular}{|c|c|c|}
\hline & \multicolumn{2}{|c|}{ BAL(25) } \\
\hline $\begin{array}{c}\text { Anaerobic } \\
\text { organism }\end{array}$ & $\begin{array}{c}\text { Lung abscess } \\
(10)\end{array}$ & $\begin{array}{c}\text { lung tumor } \\
(15)\end{array}$ \\
\hline Prevotella & $3(60 \%)$ & $\begin{array}{c}\text { Number } \\
(\%) \\
2(13.3 \%)\end{array}$ \\
\hline Veillonella & $2(40 \%)$ & $0(0 \%)$ \\
\hline Probionobacterium & $0(0 \%)$ & $1(50 \%)$ \\
\hline Lactobacillus & 0 & $1(50 \%)$ \\
\hline No growth & 5 & 13 \\
\hline
\end{tabular}

All the identified anaerobic isolates were sensitive to meropenem and Amoxicillin/clavulanic all isolates 
were sensitive to this drug. Clindamycin was effective on Lactobacillus and Veillonella (100\%). Prevotella and Propionobacterium isolates showed $66.6 \%$ and $100 \%$ resistance to clindamycin respectively. As regards the resistance of the isolates to metronidazole, both Lactobacilli and Probionobacterium isolates were resistance to metronidazole. Table(2).

Table (2) : Antimicrobial Susceptibility

\begin{tabular}{|l|c|c|c|c|c|c|c|c|}
\hline Antibiotic & \multicolumn{2}{|c|}{ Meropenem } & \multicolumn{2}{c|}{ Amoxicillin-clav } & \multicolumn{2}{c|}{ Clindamycin } & \multicolumn{2}{c|}{ Metronidazole } \\
& $\mathrm{S}$ & $\mathrm{R}$ & $\mathrm{S}$ & $\mathrm{R}$ & $\mathrm{S}$ & $\mathrm{R}$ & $\mathrm{S}$ & $\mathrm{R}$ \\
\hline Prevotella(3) & 3 & 0 & 3 & 0 & 1 & 2 & 3 & 0 \\
& $(100 \%)$ & $(0 \%)$ & $(100 \%)$ & $(0 \%)$ & $(33.3 \%)$ & $(66.6 \%)$ & $(100 \%)$ & $(0 \%)$ \\
\hline Veillonella (2) & 2 & 0 & 2 & 0 & 2 & 0 & 2 & 0 \\
& $(100 \%)$ & $(0 \%)$ & $(100 \%)$ & $(0 \%)$ & $(100 \%)$ & $(0 \%)$ & $(100 \%)$ & $(0 \%)$ \\
\hline Lactobacillus(1) & 1 & 0 & 1 & 0 & 1 & 0 & 0 & 1 \\
& $(100 \%)$ & $(0 \%)$ & $(100 \%)$ & $(0 \%)$ & $(100 \%)$ & $(0 \%)$ & $(0 \%)$ & $(100 \%)$ \\
\hline Propionibacterium & 1 & 0 & 1 & 0 & 0 & 1 & 0 & 1 \\
$(1)$ & $(100 \%)$ & $(0 \%)$ & $(100 \%)$ & $(0 \%)$ & $(0 \%)$ & $(100 \%)$ & $(0 \%)$ & $(100 \%)$ \\
\hline
\end{tabular}

Prevotellaspp. are members of the oral, vaginal,

\section{Discussion}

Anaerobic bacteria are relatively frequent pathogens in pulmonary infections including aspiration pneumonitis, lung abscess, necrotizing pneumonia and empyema [1].

The isolated anaerobic organism from BAL specimens were 7 out of 25(28\%). From lung abscess 5 cases yielded anaerobic organisms (50\%) while in cases of lung tumor only two anaerobic isolates. The anaerobic isolates were Prevotella(3), Veillonella (2), Lactobacillus (1) and Propionibacterium (1). Prevotella is predominant isolated anaerobes, 3 out of 7 with incidence $42.8 \%$. and gut microbiota and are often recovered from anaerobic infections of the respiratory tract. These infections include aspiration pneumonia, lung abscess, pulmonary empyema, and chronic otitis media and sinusitis [5].

Most of anaerobic lung infections involve multiple bacterial species, and approximately half of the patients have anaerobic bacteria combined with potentially pathogenic aerobic or facultative anaerobes [6].

A somewhat unique feature of anaerobic lung infections is the proclivity for necrosis of tissue, resulting in abscess formation or a bronchopleural fistula associated with empyema [7].

In our study; the frequency of anaerobic organism among lung abscess was 5out of 10 (50\%). Isolated 
organisms were Prevotella and vellionella. In contrast; the study done by Wang and colleagues [8] who reported that incidence of anaerobic organism in lung abscess was 39\%. Mori and colleagues [9] found that anaerobic incidence in lung abscess was 45\%. According to Takayanagi and colleagues [10] it was $26 \%$. On the other hand other studies show incidence which more higher $93 \%$ by Bartlett [7] and $100 \%$ by De and colleagues [11].

Lung abscess caused by anaerobic or mixed bacterial infection of the lower respiratory tract [12]. Prevotella and Vellionella and other anaerobic organism isolated from lung abscess according to by De and colleagues [11]. and Mori and colleagues [9]. The differences in the design of these studies such as sample size and method of identification may account for the variation in incidence of isolated organisms.

All the identified anaerobic isolates were susceptible to meropenem and amoxicillin/clavulanic . Anaerobic bacteria susceptible to meropenem, which is used in the treatment of mixed aerobic and anaerobic pulmonary infection [13]. Carbapenems possess excellent activity against aerobic and anaerobic bacteria and are often administered in serious infections[14].

In the present study, 2 out of 3 Prevotella isolates were resistant to clindamycin $66.6 \%$. Sherrard and colleagues [15] reported that all Prevotella isolates in their study were susceptible to meropenem, 56\% were clindamycin resistant, $5 \%$ were metronidazole resistant and 20\% were resistant to amoxicillin/clavulanic.

Clindamycin was considered the drug of choice for the treatment of anaerobic infections but with the emergence of resistance among Prevotella spp. (10$40 \%$ ), other related anaerobic Gram-negative bacteria
( $10 \%)$ and Peptostreptococcus spp ( $10 \%)$, this drug lost its significance as a first-line drug [16].

In the present study, both Lactobacilli and Probionobacterium isolates were resistant to metronidazole. Khassebaf and coleagues [17] reported Propionobacterium resistance $100 \%$ to metronidazole and $10 \%$ to clindamycin. On the other hand Hassan and colleagues [18] reported high Propionobacterium resistance to clindamycin (70\%).

Metronidazole resistance is common in many Grampositive anaerobic rods (Actinomyces spp., Propionibacterium spp., Lactobacillus spp.), while the prevalence of resistant Gram-positive cocci and Gram-negatives is usually very low $(<1 \%)[19]$.

\section{CONCLUSION}

In conclusion anaerobic bacteria were the most overlooked bacterial pathogens of the lower respiratory tract. They were often infrequently recovered from this site probably because of improper specimen collection and lack of appropriate anaerobic identification techniques. Anaerobic microorganisms are now widely accepted as significant pathogens in human diseases, as such, the proper diagnosis and treatment of these infections are important healthcare priorities. The emergence of antimicrobial resistance in anaerobic bacteria is a discernible phenomenon, which deserved the attention of people working in diagnostic microbiology and infectious disease treatment.

\section{REFERENCES}

[1]. Bartlett J.G, (2012):Anaerobic bacterial infection of the Lung. Anaerobe. Volume 18, Issue 2, April2012, Pages235-239.

[2]. Brook I. and Frazier.H. (1993): Aerobic and anaerobic microbiology of empyema. A 
retrospective review in two military hospitals Chest, 103 (1993), pp. 1502-1507.

[3]. Mikamo H., Arakawa S., Fujiwara M., Funada H., et al. (2011): Anaerobic infections (individual fields): respiratory infections. Japanese Society of Chemotherapy Committee on guidelines for treatment of anaerobic infections; Japanese Association for Anaerobic Infection Research. J Infect Chemother. 2011 Jul;17 Suppl 1:42-6. doi: 10.1007/s10156-0100137-6.

[4]. Clinical and Laboratory Standards Institute (CLSI) (2017: Available online: https://clsi.org/standards/products/ microbiology/ (accessed on 10 September 2017).

[5]. Tanaka S., Yoshida M., Murakami Y., et al. (2008): "The relationship of Prevotella intermedia, Prevotella nigrescens and Prevotella melaninogenica in the supragingival plaque of children, caries and oral malodor". J Clin Pediatr Dent. 32 (3): 195-200.

[6]. Enfield K.B. and Sifri C.D. (2017): Aspiration, Empyema, Lung Abscesses, and Anaerobic Infections. Fishman's Pulmonary Diseases and Disorders, 5edition Chapter 127 .McGraw-Hill Medical.

[7]. Bartlett J.G. (1993): Anaerobic bacterial infections of the lung and pleural space Clin Infect Dis, 16 (Suppl. 4) (1993), pp. S248-S255.

[8]. Wang J.L., Chen K.Y. , Fang C.T., Hsueh P.R. , Yang P.C., Chang S.C. (2005):Changing bacteriology of adult community-acquired lung abscess in Taiwan: Klebsiella pneumoniae versus anaerobesClin Infect Dis, 40 (2005), pp. 915-922.

[9]. Mori T., Ebe T., Takahashi M., Isonuma H, Ikemoto H. and Oguri T. (1993): Lung abscess: analysis of 66 cases from 1979 to 1991.Intern Med, 32 (1993), pp. 278-284.
[10]. Takayanagi N., Kagiyama N., Ishiguro T., Tokunaga D. and SugitaY. (2010): Etiology and outcome of community-acquired lung abscess. Respiration, 80 (2010), pp. 98-105.

[11]. De A., Varaiya A. and Mathur M. (2002):Anaerobes in pleuropulmonary infections Indian J Med Microbiol, 20 (2002), pp. 150-152.

[12]. 12-Kuhajda I., Zarogoulidis K., Tsirgogianni K., Tsavlis D., et al., (2015): Lung abscess-etiology, diagnostic and treatment options. Ann Transl Med. 3(13): 183.doi: 10.3978/j.issn.23055839.2015.07.08.

[13]. Blumer J.L., Saiman L., Konstan M.W. and Melnick D. (2007): The efficacy and safety of meropenem and tobramycin vsceftazidime and tobramycin in the treatment of acute pulmonary exacerbations in patients with chronic chest lesions. Chest 2007;128:233-2346.

[14]. Snydman D.R., Jacobus N.V., McDermott L.A., Golan Y., et al. (2011): Update on resistance of Bacteroides fragilis group and related species with special attention to carbapenems 20062009.Anaerobe, 17 (2011), pp. 147-151.

[15]. Sherrard L.J., Graham K.A., McGrath S.J., McIlreavey L., Hatch J., et al., (2013): Antibiotic resistance in Prevotella species isolated from patients with cystic fibrosis. J Antimicrob Chemother. 2013 Oct; 68(10): 2369-2374.

[16]. Gupta A., Vlamakis H., Shoemaker N. and Salyers A.A. (2003): A new Bacteroides conjugative transposon that carries an ermB gene. Appl. Environ. Microbiol. 2003, 69, 64556463

[17]. Khassebaf J., Hellmark B., Davidsson S., Unemo M., Nilsdotter-Augustinsson Å. and Söderquist B. (2015): Antibiotic susceptibility of Propionibacterium acnes isolated from 
orthopaedic implant-associated infections. Anaerobe. 2015 Apr;32:57-62.

[18]. Hassan I.A., Hassan M.A., Embarek M.S., Attallah D.A., El Mokhtar M.A., Alaa Eldin G.M. (2015): Antibiotic Susceptibility Patterns of Propionibacterium Acnes Isolated from Acne Vulgaris in Assiut University Hospitals, Egypt Egyptian Journal of Medical Microbiology Volume 24 / No. 4 / October 2015 67-7.

[19]. Lewis K. (2013): Platforms for antibiotic discovery. Nat. Rev. Drug Discov. 2013, 12, 371-387.

\section{Cite this article as :}

Ahmed M. Wahba, Mona A. Abdel-Messih, Randa S. Mohamed, Naglaa A. Radi, "Detection of Anaerobic Bacteria in Bronchoalveolar Lavage of Patients with Chronic Chest Lesion", International Journal of Scientific Research in Science and Technology (IJSRST), Online ISSN : 2395-602X, Print ISSN : 2395-6011, Volume 6 Issue 4, pp. 345-350, JulyAugust 2019. Available at doi : https://doi.org/10.32628/IJSRST196469 Journal URL : http://ijsrst.com/IJSRST196469 